PROCEEDINGS OF THE

AMERICAN MATHEMATICAL SOCIETY

Volume 131, Number 8, Pages 2537-2542

S 0002-9939(02)06825-9

Article electronically published on November 13, 2002

\title{
EVERY SET OF FINITE HAUSDORFF MEASURE IS A COUNTABLE UNION OF SETS WHOSE HAUSDORFF MEASURE AND CONTENT COINCIDE
}

\author{
RICHARD DELAWARE
}

(Communicated by David Preiss)

\begin{abstract}
A set $E \subseteq \mathbb{R}^{n}$ is $h$-straight if $E$ has finite Hausdorff $h$-measure equal to its Hausdorff $h$-content, where $h:[0, \infty) \rightarrow[0, \infty)$ is continuous and non-decreasing with $h(0)=0$. Here, if $h$ satisfies the standard doubling condition, then every set of finite Hausdorff $h$-measure in $\mathbb{R}^{n}$ is shown to be a countable union of $h$-straight sets. This also settles a conjecture of Foran that when $h(t)=t^{s}$, every set of finite $s$-measure is a countable union of $s$-straight sets.
\end{abstract}

\section{INTRODUCTION}

In 7], Foran introduced the notion of an $s$-straight set, that is, a set whose Hausdorff $s$-measure and Hausdorff $s$-content are equal (see Definitions 1 and 2 below). In [1] and 2], we continued the first analysis of such sets, among other results proving that in $\mathbb{R}^{2}$ a quarter circle is the countable union of 1-straight sets, verifying a conjecture of Foran. In [3] and 4, by different detailed arguments we extended that result, proving that in $\mathbb{R}^{2}$ the graphs of any convex, continuously differentiable, absolutely continuous, or increasing continuous functions, as well as regular sets of finite 1-measure, all consist of such countable unions.

Here we prove the more general result that if a function $h$ satisfies the standard doubling condition (Condition 1), then every set of finite $h$-measure in $\mathbb{R}^{n}$ is a countable union of $h$-straight sets (Definition 2, Theorem 4, and Theorem 5). This also settles the conjecture of Foran [7] that every set of finite $s$-measure is a countable union of $s$-straight sets.

Let $d$ be the standard distance function on $\mathbb{R}^{n}$ where $n \geq 1$. The diameter of an arbitrary nonempty set $U \subseteq \mathbb{R}^{n}$ is defined by $|U|=\sup \{d(x, y): x, y \in U\}$, with $|\emptyset|=0$. Given $0<\delta \leq \infty$, let $C_{\delta}^{n}$ represent the collection of subsets of $\mathbb{R}^{n}$ with diameter less than $\delta$. Let $h:[0, \infty) \rightarrow[0, \infty)$ be continuous and non-decreasing, with $h(0)=0$. (In Condition 1 we restrict $h(t)$ further.)

Definition 1 ([6, 2.10.1] or [8, p. 60]). For $E \subseteq \mathbb{R}^{n}$, let

$$
\mathcal{H}_{\delta}^{h}(E)=\inf \left\{\sum h\left(\left|E_{i}\right|\right): E \subseteq \bigcup E_{i} \text { where } E_{i} \in C_{\delta}^{n} \text { for } i=1,2, \ldots\right\} .
$$

Received by the editors August 17, 2001 and, in revised form, March 27, 2002.

2000 Mathematics Subject Classification. Primary 28A78, 28A05, 28A12.

Key words and phrases. $h$-straight, Hausdorff measure, Hausdorff content. 
Then $\mathcal{H}_{\infty}^{h}(E)$ is called the Hausdorff $h$-content of $E$, and $\mathcal{H}^{h}(E)=\sup _{\delta>0} \mathcal{H}_{\delta}^{h}(E)$ is called the Hausdorff $h$-measure, or just the $h$-measure of $E$.

Note that Hausdorff $h$-measure is Borel regular [6, 2.10.2 (1)]. Also [8, p. 54], since for any $0<\alpha<\beta \leq \infty$ it follows that $\mathcal{H}_{\beta}^{h}(E) \leq \mathcal{H}_{\alpha}^{h}(E)$, we always have the inequality

$$
\mathcal{H}_{\infty}^{h}(E) \leq \mathcal{H}^{h}(E)
$$

Definition 2. Define a set $E \subseteq \mathbb{R}^{n}$ to be $h$-straight if

$$
\mathcal{H}_{\infty}^{h}(E)=\mathcal{H}^{h}(E)<\infty
$$

A set which is the countable union of $h$-straight sets is called $\sigma h$-straight. (When $h(t)=t^{s}$, the terms are $s$-straight and $\sigma s$-straight, [1] [2].)

In 7], Foran proves the following theorem for the case of Hausdorff $s$-measure providing a useful equivalent definition of an $s$-straight set. We make the easy extension of his proof to $h$-straight sets.

Theorem 1. Let $E \subseteq \mathbb{R}^{n}$ have finite h-measure. Then, $E$ is h-straight if and only if for each $A \subseteq E$

$$
\mathcal{H}^{h}(A) \leq h(|A|)
$$

In particular, sets of zero h-measure are h-straight.

Proof. (Based on [7 p. 733].) On the one hand, suppose for each $A \subseteq E$ that $\mathcal{H}^{h}(A) \leq h(|A|)$. Then

$$
\begin{aligned}
\mathcal{H}^{h}(E) & \geq \mathcal{H}_{\infty}^{h}(E)=\inf \left\{\sum h\left(\left|E_{i}\right|\right): E=\bigcup E_{i}\right\} \\
& \geq \inf \left\{\sum \mathcal{H}^{h}\left(E_{i}\right): E=\bigcup E_{i}\right\} \\
& \geq \mathcal{H}^{h}(E),
\end{aligned}
$$

where the infima are over countable covers $\left\{E_{i}\right\}$ of $E$. Since $E$ has finite $h$-measure, it then follows that $\mathcal{H}_{\infty}^{h}(E)=\mathcal{H}^{h}(E)<\infty$. Hence $E$ is $h$-straight. Conversely, suppose $\mathcal{H}_{\infty}^{h}(E)=\mathcal{H}^{h}(E)<\infty$. If there were a subset $A \subseteq E$ such that $\mathcal{H}^{h}(A)>$ $h(|A|)$, then, since $h$ is non-decreasing and $\mathcal{H}_{\infty}^{h}(E \backslash A) \leq \overline{\mathcal{H}^{h}}(E \backslash A) \leq \mathcal{H}^{h}(E)<\infty$, we have

$$
\begin{aligned}
\mathcal{H}^{h}(E) & =\mathcal{H}^{h}(A)+\mathcal{H}^{h}(E \backslash A) \\
& >h(|A|)+\mathcal{H}_{\infty}^{h}(E \backslash A) \\
& \geq \mathcal{H}_{\infty}^{h}(A)+\mathcal{H}_{\infty}^{h}(E \backslash A) \\
& \geq \mathcal{H}_{\infty}^{h}(E),
\end{aligned}
$$

contradicting the assumption that $\mathcal{H}_{\infty}^{h}(E)=\mathcal{H}^{h}(E)$.

Theorem 22 is proved in 1], 2], for $s$-straight sets using a standard exhaustion argument. The proof of the generalization here to $h$-straight sets is omitted.

Theorem 2. Let $E \subseteq \mathbb{R}^{n}$ have finite h-measure. Then, every $\mathcal{H}^{h}$-measurable subset of positive h-measure of $E$ contains an $h$-straight set of positive $h$-measure if and only if $E$ is $\sigma$ h-straight. 


\section{MAIN RESUlT}

To prepare for the proof of the main result, Theorem 4 we begin with a wellknown definition.

Definition 3 ([5, p. 21]). Let $E \subseteq \mathbb{R}^{n}$ have finite $h$-measure, and $x \in E$. The upper convex density of $E$ at $x$ is defined to be

$$
\bar{D}_{c}^{h}(E, x)=\lim _{\eta \rightarrow 0} \sup \left\{\frac{\mathcal{H}^{h}(E \cap S)}{h(|S|)}: x \in S \text { and } 0<|S| \leq \eta\right\}
$$

where the supremum is over all convex sets $S \subseteq \mathbb{R}^{n}$.

Note that since any set is contained in a convex set of the same diameter, Definition 3 may be interpreted as taking the supremum over all sets $S \subseteq \mathbb{R}^{n}$. We also assume $h$ satisfies the following standard doubling condition.

Condition 1. For some $c<\infty, \quad h(2 t) \leq c \cdot h(t)$ for all $t>0$.

Theorem 3 is the restriction we need here of a result of Federer, [6, 2.10.18 (3)].

Theorem 3 (Corollary to [6, 2.10.18 (3)]). Let $E \subseteq \mathbb{R}^{n}$ have finite h-measure, where $h$ satisfies Condition 1 . Then for $\mathcal{H}^{h}$-almost all $x \in E$ it follows that

$$
\bar{D}_{c}^{h}(E, x) \leq 1 .
$$

Lemma1 is then a straightforward consequence of Theorem3. The proof, though a standard argument, is included for completeness.

Lemma 1. Let $E \subseteq \mathbb{R}^{n}$ have finite h-measure, where $h$ satisfies Condition 1. Let $\left\{\varepsilon_{j}\right\}_{j=0}^{\infty}$ be a positive decreasing sequence of real numbers such that $\lim _{j \rightarrow \infty} \varepsilon_{j}=0$. Then there exists $A \subseteq E$ with positive h-measure satisfying the condition that for each $j \geq 0$ there is $\rho_{j}>0$ so that for all $S \subseteq \mathbb{R}^{n}$ with $0<|S| \leq \rho_{j}$ we have

$$
\mathcal{H}^{h}(A \cap S) \leq\left(1+\varepsilon_{j}\right) \cdot h(|S|) .
$$

Proof. Suppose $h$ is such a function, $E \subseteq \mathbb{R}^{n}$ satisfies $\mathcal{H}^{h}(E)<\infty$, and $\left\{\varepsilon_{j}\right\}_{j=0}^{\infty}$ is a positive decreasing sequence of real numbers such that $\lim _{j \rightarrow \infty} \varepsilon_{j}=0$. By Theorem 3, for $\mathcal{H}^{h}$-almost all $x \in E$ it follows that

$$
\bar{D}_{c}^{h}(E, x)=\lim _{\eta \rightarrow 0} \sup \left\{\frac{\mathcal{H}^{h}(E \cap S)}{h(|S|)}: x \in S \text { and } 0<|S| \leq \eta\right\} \leq 1 .
$$

So given $\varepsilon_{j}>0$ there exists a corresponding $\eta_{j}(x)>0$ such that

$$
\sup \left\{\frac{\mathcal{H}^{h}(E \cap S)}{h(|S|)}: x \in S \text { and } 0<|S| \leq \eta_{j}(x)\right\} \leq 1+\varepsilon_{j} .
$$

Then for each $S \subseteq \mathbb{R}^{n}$ with $x \in E \cap S$ and $0<|S| \leq \eta_{j}(x)$ we have

$$
\mathcal{H}^{h}(E \cap S) \leq\left(1+\varepsilon_{j}\right) \cdot h(|S|)
$$

Now let $\left\{\rho_{j}\right\}_{j=0}^{\infty}$ be a positive decreasing sequence of real numbers such that $\lim _{j \rightarrow \infty} \rho_{j}=0$. Define

$$
A_{j}=\left\{x \in \mathbb{R}^{n}: \text { If } x \in S \text { and } 0<|S| \leq \rho_{j} \text {, then } \mathcal{H}^{h}(E \cap S) \leq\left(1+\varepsilon_{j}\right) \cdot h(|S|)\right\} .
$$

Then $A_{j}$ is a Borel (in fact, closed) set, hence $\mathcal{H}^{h}$-measurable. Since $\bar{D}_{c}^{h}(E, x) \leq 1$ for $\mathcal{H}^{h}$-almost all $x \in E$, we may further choose $\rho_{j}$ small enough so that given $\delta$ satisfying $0<\delta<\mathcal{H}^{h}(E)$, we have $\mathcal{H}^{h}\left(E \backslash A_{j}\right)<\frac{\delta}{2^{j+1}}$. Hence, $\sum_{j=0}^{\infty} \mathcal{H}^{h}\left(E \backslash A_{j}\right)<$ 
$\sum_{j=0}^{\infty} \frac{\delta}{2^{j+1}}=\delta$. For such choices of $\rho_{j}$, define $A=\bigcap_{j=0}^{\infty} A_{j}$. By the definition of $A$ as an intersection, for each $j \geq 0$ there is $\rho_{j}>0$, so for all $S \subseteq \mathbb{R}^{n}$ with $0<|S| \leq \rho_{j}$ we have that $A$ satisfies

$$
\mathcal{H}^{h}(A \cap S) \leq \mathcal{H}^{h}(E \cap S) \leq\left(1+\varepsilon_{j}\right) \cdot h(|S|) .
$$

Also, $A$ has positive $h$-measure since

$$
\begin{aligned}
\mathcal{H}^{h}(A) & =\mathcal{H}^{h}\left(\bigcap_{j=0}^{\infty} A_{j}\right)=\mathcal{H}^{h}(E)-\mathcal{H}^{h}\left(\bigcup_{j=0}^{\infty}\left(E \backslash A_{j}\right)\right) \\
& \geq \mathcal{H}^{h}(E)-\sum_{j=0}^{\infty} \mathcal{H}^{h}\left(E \backslash A_{j}\right)>\mathcal{H}^{h}(E)-\delta>0 .
\end{aligned}
$$

So, $A \subseteq E$ is the desired set.

The argument used in the proof of Theorem 4 below was developed from a suggestion of Preiss [10] for sets of finite $s$-measure made after reading a preprint of [3].

Theorem 4. If $E \subseteq \mathbb{R}^{n}$ has finite h-measure where $h$ satisfies Condition 1 , then there exists $B \subseteq E$ with positive h-measure such that $B$ is h-straight.

Proof. Let $E \subseteq \mathbb{R}^{n}$ have finite $h$-measure, where $h$ is such a function. Let $\left\{\varepsilon_{j}\right\}_{j=0}^{\infty}$ be a positive, decreasing sequence of real numbers such that $\sum_{j=0}^{\infty} \varepsilon_{j}<1$. Hence, $\lim _{j \rightarrow \infty} \varepsilon_{j}=0$. So, by Lemma 1 there exists $A \subseteq E$ with positive $h$-measure and corresponding $\rho_{j}>0$ such that for all $S \subseteq \mathbb{R}^{n}$ with $0<|S| \leq \rho_{j}$ we have

$$
\mathcal{H}^{h}(A \cap S) \leq\left(1+\varepsilon_{j}\right) \cdot h(|S|) .
$$

For $j \geq 0$ replace as needed the $\rho_{j}$ with values $r_{j}$ small enough so that $\lim _{j \rightarrow \infty} r_{j}=$ 0 , with both $2 r_{0}<\rho_{0}$ and

$$
2 r_{j+1}<\min \left(r_{j}, \rho_{j+1}\right) .
$$

By the continuity of $h$ we may also assume the $r_{j}$ small enough so that whenever $r_{j} \leq t \leq r_{j-1}$, we have

$$
h\left(t+2 r_{j+1}\right)<\left(1+\varepsilon_{j-1}^{2}\right) \cdot h(t) .
$$

We may further assume that

$$
0<\mathcal{H}^{h}(A) \leq h\left(r_{0}\right)
$$

by the continuity of $h$-measure, since $0<\mathcal{H}^{h}(A) \leq \mathcal{H}^{h}(E)<\infty$.

Now, for each fixed $j \geq 1$, let $\left\{A_{j, i}\right\}_{i}$ be a partition of $A$ into (relatively) Borel subsets such that $\left|A_{j, i}\right|<r_{j+1}$ for every $i$. Then, for $j \geq 1$ let $B_{j, i} \subseteq A_{j, i}$ be slightly smaller in measure, satisfying

$$
\mathcal{H}^{h}\left(B_{j, i}\right)=\left(1-\varepsilon_{j-1}\right) \cdot \mathcal{H}^{h}\left(A_{j, i}\right) .
$$

It then follows for each fixed $j \geq 1$ that since the $A_{j, i}$ partition $A$,

$$
\mathcal{H}^{h}\left(\bigcup_{i} B_{j, i}\right)=\sum_{i} \mathcal{H}^{h}\left(B_{j, i}\right)=\left(1-\varepsilon_{j-1}\right) \cdot \sum_{i} \mathcal{H}^{h}\left(A_{j, i}\right)=\left(1-\varepsilon_{j-1}\right) \cdot \mathcal{H}^{h}(A) .
$$


Define $B=\bigcap_{j \geq 1} \bigcup_{i} B_{j, i}$. Observe that using the subadditivity of $\mathcal{H}^{h}$, we have

$$
\begin{aligned}
\mathcal{H}^{h}(A \backslash B) & =\mathcal{H}^{h}\left(\bigcup_{j \geq 1}\left[A \backslash \bigcup_{i} B_{j, i}\right]\right) \\
& \leq \sum_{j \geq 1}\left[\mathcal{H}^{h}(A)-\mathcal{H}^{h}\left(\bigcup_{i} B_{j, i}\right)\right] \\
& =\left(\sum_{j \geq 1} \varepsilon_{j-1}\right) \cdot \mathcal{H}^{h}(A)<\mathcal{H}^{h}(A) .
\end{aligned}
$$

Thus $\mathcal{H}^{h}(B)=\mathcal{H}^{h}(A)-\mathcal{H}^{h}(A \backslash B)>0$, so $B$ has positive $h$-measure. Using Theorem 1 we show that $B$ is the desired $h$-straight subset of $E$ by showing that $\mathcal{H}^{h}(B \cap S) \leq h(|S|)$ for every $S \subseteq \mathbb{R}^{n}$.

First, for any $S \subseteq \mathbb{R}^{n}$ such that $|S|>r_{0}$, since $B \cap S \subseteq A$ and $h$ is non-decreasing we immediately have that

$$
\mathcal{H}^{h}(B \cap S) \leq \mathcal{H}^{h}(A) \leq h\left(r_{0}\right) \leq h(|S|) .
$$

So, consider all other sets $S \subseteq \mathbb{R}^{n}$ such that $r_{j}<|S| \leq r_{j-1}$ for some $j \geq 1$. Define

$$
S^{\prime}=\left\{x \in \mathbb{R}^{n}: d(x, S) \leq r_{j+1}\right\} .
$$

So $S \subseteq S^{\prime}$. By the restrictions on $\left\{r_{k}\right\}_{k=0}^{\infty}$ we then have that

$$
0<\left|S^{\prime}\right| \leq|S|+2 r_{j+1}<r_{j-1}+r_{j}<2 r_{j-1}<\rho_{j-1} .
$$

By definition for each $j \geq 1$ we have $B \cap A_{j, i} \subseteq B_{j, i}$. Denote by $\bigcup^{\prime}$ the union and by $\sum^{\prime}$ the summation over those indices $i$ for which $S \cap B_{j, i} \neq \emptyset$. So for $j \geq 1$ we have $B \cap S \subseteq \bigcup_{i}^{\prime} B_{j, i}$. Also, because $\left|A_{j, i}\right|<r_{j+1}$ it follows that each $A_{j, i} \subseteq A \cap S^{\prime}$ for those same indices $i$. Then, for any fixed $j \geq 1$, we have

$$
\begin{aligned}
\mathcal{H}^{h}(B \cap S) & \leq \sum_{i}^{\prime} \mathcal{H}^{h}\left(B_{j, i}\right) \\
& =\left(1-\varepsilon_{j-1}\right) \cdot \sum_{i}^{\prime} \mathcal{H}^{h}\left(A_{j, i}\right)=\left(1-\varepsilon_{j-1}\right) \cdot \mathcal{H}^{h}\left(\bigcup_{i}^{\prime} A_{j, i}\right) \\
& \leq\left(1-\varepsilon_{j-1}\right) \cdot \mathcal{H}^{h}\left(A \cap S^{\prime}\right) .
\end{aligned}
$$

Since $r_{j}<|S| \leq r_{j-1}$ and $h$ is non-decreasing, again by the restrictions on $\left\{r_{k}\right\}_{k=0}^{\infty}$ we have

$$
h\left(\left|S^{\prime}\right|\right) \leq h\left(|S|+2 r_{j+1}\right)<\left(1+\varepsilon_{j-1}^{2}\right) \cdot h(|S|) .
$$

Since also $0<\left|S^{\prime}\right|<\rho_{j-1}$, by inequality $(*)$ we further have

$$
\mathcal{H}^{h}\left(A \cap S^{\prime}\right) \leq\left(1+\varepsilon_{j-1}\right) \cdot h\left(\left|S^{\prime}\right|\right) .
$$

So, by inequality $(* *)$, we finally conclude

$$
\begin{aligned}
\mathcal{H}^{h}(B \cap S) & \leq\left(1-\varepsilon_{j-1}\right) \cdot\left(1+\varepsilon_{j-1}\right) \cdot h\left(\left|S^{\prime}\right|\right) \\
& <\left(1-\varepsilon_{j-1}^{2}\right) \cdot\left(1+\varepsilon_{j-1}^{2}\right) \cdot h(|S|) \\
& =\left(1-\varepsilon_{j-1}^{4}\right) \cdot h(|S|) \\
& <h(|S|) .
\end{aligned}
$$


Therefore in particular if $S \subseteq B$ it follows that $\mathcal{H}^{h}(S)=\mathcal{H}^{h}(B \cap S) \leq h(|S|)$. So by Theorem [ the set $B$ is $h$-straight with positive $h$-measure, as desired.

Theorem 5. If $E \subseteq \mathbb{R}^{n}$ has finite h-measure where $h$ satisfies Condition 1 , then $E$ is $\sigma$ h-straight.

Proof. Let $E \subseteq \mathbb{R}^{n}$ have finite $h$-measure, where $h$ is such a function. By Theorem 4 every $\mathcal{H}^{h}$-measurable subset of $E$ of positive $h$-measure contains an $h$-straight subset of positive $h$-measure. By Theorem 2 it then follows that $E$ is $\sigma h$-straight.

\section{ACKNOWLEDGEMENTS}

The author thanks James Foran for helpful conversations, and David Preiss [10], Pertti Mattila [9, and the referee for valuable suggestions and improvements.

\section{REFERENCES}

[1] R. Delaware, Sets Whose Hausdorff Measure Equals Method I Outer Measure, Ph.D. Dissertation, University of Missouri-Kansas City, 2000.

[2] R. Delaware, Sets Whose Hausdorff Measure Equals Method I Outer Measure, Real Anal. Exchange, 27(2), 2001/2, 535-562.

[3] R. Delaware, Graphs of Convex Functions are $\sigma 1$-straight, Rocky Mountain Journal of Mathematics, to appear.

[4] R. Delaware and L. Eifler, Graphs of Functions, Regular Sets, and s-straight Sets, Real Anal. Exchange, 26(2), 2000/1, 885-892. MR 2002e:28010

[5] K. J. Falconer, The Geometry of Fractal Sets, Cambridge University Press, 1985. MR 88d:28001

[6] H. Federer, Geometric Measure Theory, Springer-Verlag, 1969. MR 41:1976

[7] J. Foran, Measure-Preserving Continuous Straightening of Fractional Dimension Sets, Real Anal. Exchange (2), 21 (1995-96), 732-738. MR 97k:28013

[8] P. Mattila, Geometry of Sets and Measures in Euclidean Spaces, Fractals and rectifiability, Cambridge University Press, 1995. MR 96h:28006

[9] P. Mattila, private email communication, 2 April 2001.

[10] D. Preiss, private email communication, 20 October 2000.

Department of Mathematics and Statistics, HaAg Hall Room 206, University of MisSouri - Kansas City, 5100 Rockhill Rd., Kansas City, Missouri 64110

E-mail address: RDelaware3141@cs.com 